\title{
1 Spatial separation of 2-propanol monomer and its ionization-fragmentation pathways
}

\author{
$2 \quad$ Jia Wang, ${ }^{1, \mathbb{m}}$ Lanhai He, ${ }^{1, \$}$ Jovana Petrovic, ${ }^{1}$ Ahmed Al-Refaie,,${ }^{1,}{ }^{\ddagger}$ \\ $3 \quad$ Helen Bieker, ${ }^{1,2}$ Jolijn Onvlee, ${ }^{1,3}$ Karol Długołęcki, ${ }^{1}$ and Jochen Küpper ${ }^{1,2,3, *}$ \\ ${ }_{4}^{1}$ Center for Free-Electron Laser Science, Deutsches Elektronen-Synchrotron DESY, Notkestraße 85, 22607 Hamburg, Germany \\ ${ }_{5}^{2}$ Department of Physics, Universität Hamburg, Luruper Chaussee 149, 22761 Hamburg, Germany \\ 6 $\quad{ }^{3}$ Center for Ultrafast Imaging, Universität Hamburg, Luruper Chaussee 149, 22761 Hamburg, Germany
}

The spatial separation of 2-propanol monomer from its clusters in a molecular beam by an electrostatic deflector was demonstrated. Samples of 2-propanol monomer with a purity of $90 \%$ and a beam density of $7 \times 10^{6} \mathrm{~cm}^{-3}$ were obtained. These samples were utilized to study the femtosecond-laserinduced strong-field multi-photon ionization and fragmentation of 2-propanol using non-resonant $800 \mathrm{~nm}$ light with peak intensities of $3-7 \times 10^{13} \mathrm{~W} / \mathrm{cm}^{2}$.

\section{In memoriam Jon T. Hougen}

7

\section{INTRODUCTION}

8 2-propanol $\left(\mathrm{C}_{3} \mathrm{H}_{8} \mathrm{O}\right.$, also isopropyl alcohol, isopropanol) $\boldsymbol{9}$ is the simplest secondary alcohol. It possesses a structure 10 with three nonrigid internal rotations, i. e., of the hydroxyl 11 group $\mathrm{OH}$ and two methyl tops $\mathrm{CH}_{3}[1-3$. 2-propanol has 12 attracted much attention, not only because it is highly 13 valued as a preservative and used as an antiseptic in the 14 clinical environment, but also since it is widely used as 15 an industrial solvent and cleaning fluid, such as gasoline 16 additive, an alkylating agent, and a disinfectant $[4,5$. The 17 vibrational spectrum of 2-propanol has been studied in the 18 early 1960s, which suggested that 2-propanol in the gas 19 phase exists in trans and gauche conformations [6]. These 2o stable isomers were confirmed by the microwave [7-10] 21 and the millimeter- and submillimeter-wave spectra $[3,5$.

22 Supersonic molecular beam is a valuable tool for molec23 ular spectroscopy and the studies of molecular dynamics 24 and reactions [11-13]. For instance, this was exploited ${ }_{25}$ in studies of the hydrogen bonding in 2-propanol [14] 26 and its hydrogen-bonded complexes $[15,16\rfloor$. Generally, 27 the supersonic expansion provides beams of molecules at 28 low rotational temperatures. However, clusters can be 29 formed $[11,12,17]$ due to the attractive forces between so molecules. The temperature- and pressure-dependence 31 of its cluster formation was investigated [18, 19. Other 32 factors influencing the cluster formation in expanding su33 personic jets are the nozzle size and shape and the carrier 34 gas $[11,20,21$. Larger clusters are often fragile and then 35 fragment upon excitation or ionization before detection, 36 rendering size-assignment from spectra ambiguous or even 37 impossible $[22,23]$.

38 For prospective studies of 2-propanol in chemical39 reactions $[24,25$. or diffractive-imaging experiments $[26$, 4027 , a cold and pure beam of 2-propanol separated from 41 clusters as well as seed gas is necessary [28, 29]. Such 42 spatial separation of molecular conformers was previously 43 achieved using inhomogeneous electric fields $[29,30$. Dif44 ferent species of complex molecules can be spatially sepa45 rated within a cold molecular beam by the electrostatic
46 deflector 29 , which was demonstrated in a number of 47 pioneering experiments on the separation of individual 48 quantum states $31-33$, structural isomers $[34,35$, or spe49 cific cluster sizes $\mid 36-40\rfloor$. Here, the 2-propanol monomer 50 is spatially separated from the original molecular beam 51 using the deflector and the purified samples are exploited 52 in femtosecond-laser ionization studies.

\section{EXPERIMENTAL METHODS}

54 2-propanol was commercially obtained (Carl Roth ${ }_{55} \mathrm{GmbH},>99 \%$ ) and used without further purification. 56 Fig. 1 shows a schematic of the experimental setup, similar 57 to a previously described one [37. Briefly, 2-propanol at 58 room temperature is seeded in 2 bar of helium and super59 sonically expanded into vacuum through a cantilever piezo 6o valve [41] at a repetition rate of $20 \mathrm{~Hz}$. The generated 61 jet was differentially pumped and collimated by two skim62 mers, which were placed $55 \mathrm{~mm}(\varnothing=3 \mathrm{~mm})$ and $365 \mathrm{~mm}$ ${ }_{63}(\varnothing=1.5 \mathrm{~mm})$ downstream of the valve. The resulting 64 molecular beam was directed through the electrostatic de-

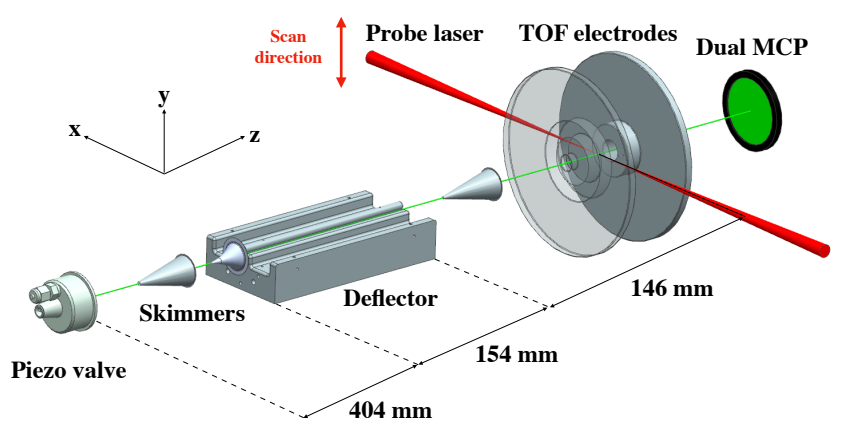

FIG. 1. Schematic of the experimental setup, consisting of a pulsed valve, the electrostatic deflector, a femtosecond-pulse laser system, and a two-plate time-of-flight (TOF) mass spectrometer; see text for details. 
${ }_{65}$ flector, which was placed $404 \mathrm{~mm}$ downstream of the valve, ${ }_{1 \mathbf{1 8}}$ suggests that $[\mathrm{M}-\mathrm{OH}]^{+}$is a major fragmentation product 66 before passing through a third skimmer $(\varnothing=1.5 \mathrm{~mm}) .119$ of these larger clusters.

67 2-propanol molecules were ionized by amplified femtosec- $120 \quad 2$-propanol is a nearly symmetric oblate rotor, the ro68 ond laser pulses with a duration of $45 \mathrm{fs}$ (full-width at 121 tational constants for trans and gauche were obtained 69 half maximum, FWHM) with a spectrum centered around ${ }_{122}$ by microwave spectroscopy [8]. The dipole-moment com$70800 \mathrm{~nm}$. By focusing the laser with pulse energies of $30 \mu \mathrm{J}_{123}$ ponents are $\mu_{a}=0, \mu_{b}=1.40 \mathrm{D}, \mu_{c}=0.73 \mathrm{D}$ for the ${ }_{71}$ and $60 \mu \mathrm{J}$ to $50 \mu \mathrm{m}$, we obtained nominal peak intensities ${ }_{124}$ trans $[7]$ and $\mu_{a}=1.114 \mathrm{D}, \mu_{b}=0.737 \mathrm{D}, \mu_{c}=0.813 \mathrm{D}$ 72 of $3 \times 10^{13} \mathrm{~W} / \mathrm{cm}^{2}$ and $7 \times 10^{13} \mathrm{~W} / \mathrm{cm}^{2}$, respectively. A 125 for the gauche conformer, respectively [8]. Their energy 73 two-plate velocity map imaging (VMI) [42] was operated ${ }_{126}$ difference is smaller than $1 \mathrm{~kJ} / \mathrm{mol}$ and the isomerization 74 as a time-of-flight (TOF) mass spectrometer (MS). Ions 127 barrier corresponding to rotation of the hydroxyl moiety 75 were counted through a single-shot detector readout and ${ }_{\mathbf{1 2 8}}$ is low 16 . The gauche conformer is more stable than the 76 centroiding algorithm [43, and summed up to yield the 129 trans conformer and there is a strong conformational re77 mass spectra shown in Fig. $2 \mathrm{a}, \mathrm{b}$

\section{RESULTS AND DISCUSSION} The mass spectra at peak intensities of $3 \times 10^{13} \mathrm{~W} / \mathrm{cm}^{2}$ 80 and $7 \times 10^{13} \mathrm{~W} / \mathrm{cm}^{2}$ of the direct $(0 \mathrm{kV})$ and deflected 13 $\mathbf{8 1}(13 \mathrm{kV})$ molecular beams are shown in Fig. 2. The spec- 137 $\mathbf{8 2}$ trum of the direct beam shows 2-propanol fragment ions $\mathbf{1 3 8}$ gauche forms at low temperatures as they are essentially
$\mathbf{8 \mathbf { 3 }}\left([\mathrm{M}-\mathrm{OH}]^{+}\right.$and $\left.\left[\mathrm{M}-\mathrm{CH}_{3}\right]^{+}\right)$, monomer ions $\mathrm{M}^{+}$, and pro- $\mathbf{1 3 9}$ identical. For the analysis in this work, we used only the 84 tonated 2-propanol and its cluster ions $\left[\mathrm{M}_{n}+\mathrm{H}\right]^{+}$up to $\mathbf{1 4 0}$ trans-conformer deflection simulations.

${ }_{\mathbf{8 5}} n=5$. Here, $\mathrm{M}$ is the parent molecule and $[\mathrm{M}-\mathrm{OH}]^{+}{ }_{14 \mathbf{1}}$ The simulated vertical molecular-beam profiles of the 86 and $\left[\mathrm{M}_{-} \mathrm{CH}_{3}\right]^{+}$specify fragments where $\mathrm{M}$ lost $\mathrm{OH}$ or $\mathbf{1 4 2}$ 2-propanol monomer are shown in Fig. 2 c and Fig. 2 d. ${ }_{87} \mathrm{CH}_{3}$, respectively. The mass spectra in Fig. 2 a and $\mathbf{1 4 3}$ The Stark energies for all rotational states up to $J=14$ $\mathbf{8 8}$ Fig. 2 b were normalized to their largest peak. Note that $\mathbf{1 4 4}$ were calculated using a basis of field-free rotational states 89 the mass spectra of the direct and deflected beams are 145 up to $J=30$ [45]. For every quantum state, $1 \times 10^{5}$ tra9o shown on different vertical scales for improved visibility. 146 jectories were calculated. The initial beam temperature 91 Larger clusters were not detected due to the recorded $\mathbf{1 4 7}$ that described the experimental observations best was 92 TOF interval in the experiments. Note that the clusters $\mathbf{1 4 8}$ determined to be 3.5(5) K. The shaded (dark red) area $\mathbf{9 3}$ of 2-propanol in the interaction region are neutral and $\mathbf{1 4 9}$ depicts the error estimate of the 2-propanol simulation 94 that the protonated-cluster ions in the mass spectrum $\mathbf{1 5 0}$ due to the temperature uncertainty.

95 resulted from the ionization detection process $\left[39,40 \mid\right.$. $\quad \mathbf{1 5}_{\mathbf{1 5}}$ In Fig. 2 c the deflected profiles (dashed lines) of 96 The spatial vertical molecular-beam-density profiles 152 monomer (red triangle up) and [M-CH$]^{+}$ions (black 97 for 2-propanol monomer ions, fragment ions, protonated 153 triangle down) matched very well over the whole deflec-

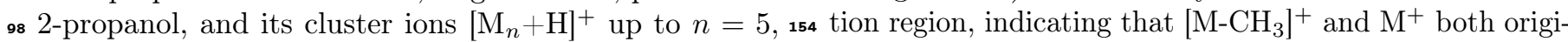
99 ionized with the laser peak intensities of $3 \times 10^{13} \mathrm{~W} / \mathrm{cm}^{2}{ }_{155}$ nated from the parent molecule. However, at higher peak 100 and $7 \times 10^{13} \mathrm{~W} / \mathrm{cm}^{2}$, are shown in Fig. $2 \mathrm{c}$ and Fig. $2 \mathrm{~d}$, 156 ionization-laser intensity, Fig. $2 \mathrm{~d}$, the profiles of monomer

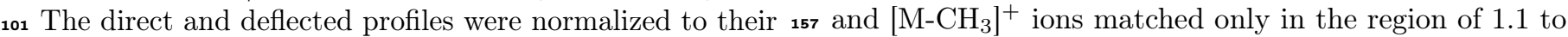
102 largest signals. For better visibility, the $\mathrm{M}^{+}$deflection 1 s8 $1.8 \mathrm{~mm}$. The higher $\left[\mathrm{M}^{-} \mathrm{CH}\right]^{+}$signal in the $[-1,1]$ regions 103 profile has been scaled up by a factor of 5.5. When a 15 indicates the contribution of larger clusters to this frag104 voltage of $13 \mathrm{kV}$ is applied to the deflector, the profiles of $\mathbf{1 6 0}$ ment in the ion signals. Nevertheless, this behavior nicely 105 fragment ions $\left([\mathrm{M}-\mathrm{OH}]^{+}\right.$and $\left.\left[\mathrm{M}-\mathrm{CH}_{3}\right]^{+}\right)$, and the parent 1 16 confirms the selection of monomers from the expansion 106 ion $\mathrm{M}^{+}$are shifted by $+1.8 \mathrm{~mm}$ at both peak intensities; 162 in the deflected part at positions larger than $1.1 \mathrm{~mm}$. 107 the $\left[2 \mathrm{M}-\mathrm{CH}_{3}\right]^{+}$profile also has a tail with significant $\mathbf{1 6 3}$ The deflected-beam mass spectra in Fig. 2 a and Fig. 2 b 108 deflection, which we ascribe to a fairly polar cluster, but 164 mainly contained peaks corresponding to [M-OH] ${ }^{+}$, [M109 this species deflects less and has only a small population $\left.{ }_{165} \mathrm{CH}_{3}\right]^{+}, \mathrm{M}^{+}$and $[2 \mathrm{M}-\mathrm{CH}]^{+}$at both laser peak intensities 110 in the beam. The protonated 2-propanol and its cluster ${ }_{\mathbf{1 6}}$ The fragments were caused by the fs-pulse ionization 111 ions in Fig. $2 \mathrm{c}$ and Fig. $2 \mathrm{~d}$ do not deflect in the region $\mathbf{1 6 7}$ process, however, no hydrogen ions were observed in the 112 of 1.1 to $1.8 \mathrm{~mm}$, which shows that dimers and larger 168 TOF spectrum in Fig. 2 a due to the low laser peak 113 clusters generally deflect much less than the monomer and $\mathbf{1 6 9}$ intensity. The ratios of different fragments and $\mathrm{M}^{+}$ions $1 \mathbf{1 4}$ the monomer ion signal originated from the monomer. In $\mathbf{1 7 0}$ from the center position to 1.6 mm are shown in Fig. 3 . 115 Fig. 2 a and Fig. 2 b the intensity of the cluster peaks is $\mathbf{1 7 1}$ For both laser peak intensities, the ratios for [M-OH] ${ }^{+}$,

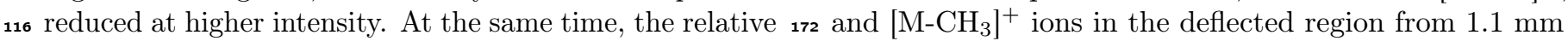
117 intensity of the $[\mathrm{M}-\mathrm{OH}]^{+}$peak is strongly increased, which ${ }_{173}$ to $1.6 \mathrm{~mm}$ are constant, which suggests $[\mathrm{M}-\mathrm{OH}]^{+}$, $[\mathrm{M}-$ 

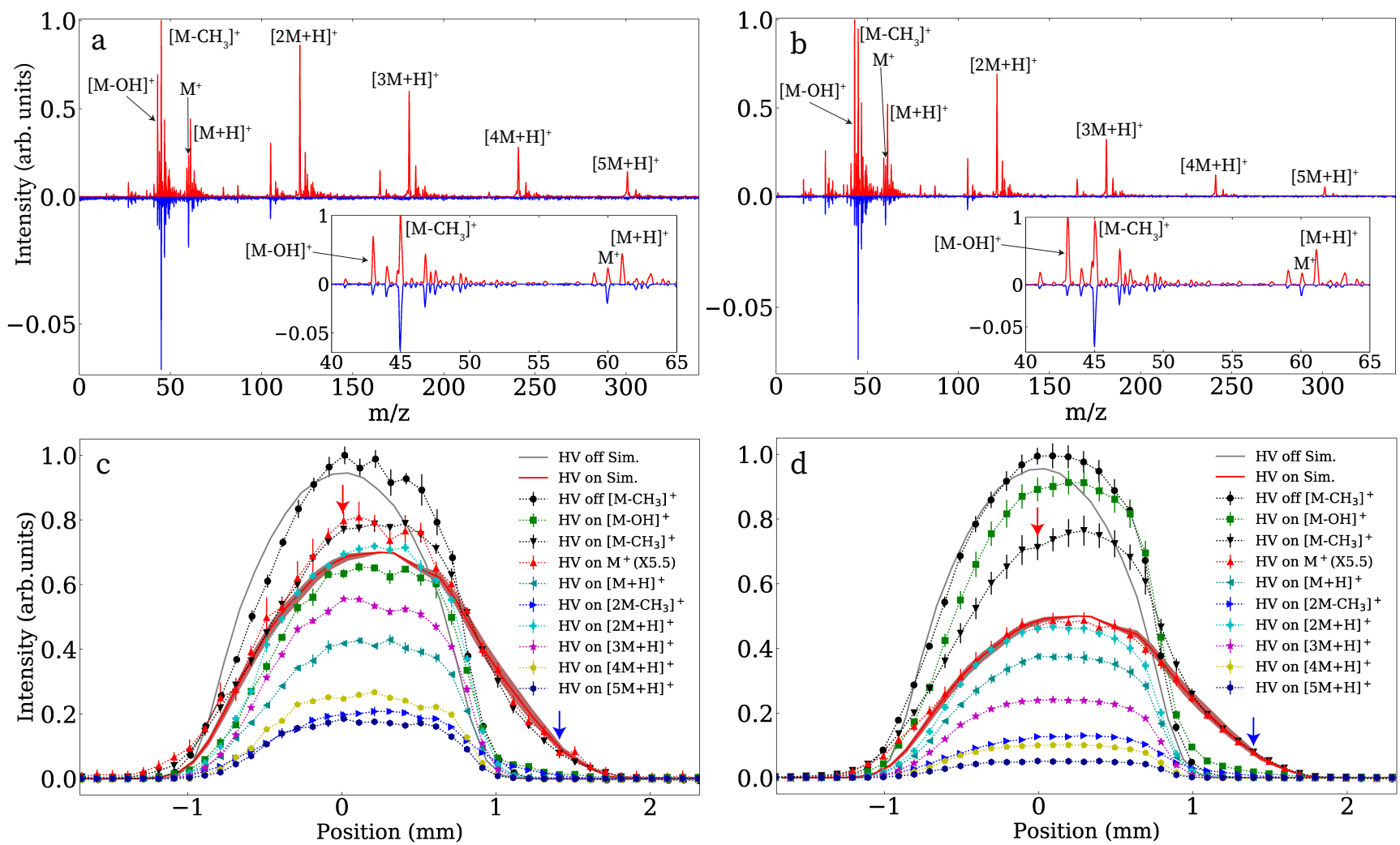

FIG. 2. Mass spectra and vertical molecular-beam-density profiles with ionization-laser peak intensities of $(\mathrm{a}, \mathrm{c}) 3 \times 10^{13} \mathrm{~W} / \mathrm{cm}^{2}$ and (b, d) $7 \times 10^{13} \mathrm{~W} / \mathrm{cm}^{2}$. (a) and (b) TOF-MS in the central part of the molecular beam with the deflector off (red) and at a position of $+1.4 \mathrm{~mm}$ with a deflection voltage of $13 \mathrm{kV}$ (blue). (c) and (d) $0 \mathrm{kV}$ (circle) and $13 \mathrm{kV}$ vertical spatial-beam-profiles for both pulse energies. In the legends $\mathrm{M}$ refers to the monomer parent ion. Dashed lines show fragment cations of 2-propanol $\left([\mathrm{M}-\mathrm{OH}]^{+}\right.$(green), $\left[\mathrm{M}-\mathrm{CH}_{3}\right]^{+}$(black), $\left[2 \mathrm{M}-\mathrm{CH}_{3}\right]^{+}$(blue)), monomer cation $\mathrm{M}^{+}$(red), protonated 2-propanol $[\mathrm{M}+\mathrm{H}]^{+}($darkcyan $)$ and its clusters up to $n=5\left([2 \mathrm{M}+\mathrm{H}]^{+}(\right.$cyan $),[3 \mathrm{M}+\mathrm{H}]^{+}$(magenta), $[4 \mathrm{M}+\mathrm{H}]^{+}$(yellow), $[5 \mathrm{M}+\mathrm{H}]^{+}($darkblue $\left.)\right)$. Simulated deflection profiles of the direct 2-propanol monomer beam (gray) as well as the deflected 2-propanol monomer beam (red) are shown as solid lines. The shaded (dark red) area depicts the error estimate of the 2-propanol simulation due to the temperature uncertainty. The red and blue arrows indicate the positions in the deflected beam where the mass spectra shown in (a) and (b) were measured.

$\left.{ }_{174} \mathrm{CH}_{3}\right]^{+}$and $\mathrm{M}^{+}$ions come from the same parent, the ${ }_{103}$ This is a nearly 22 -fold increase in the fractional density 175 monomer. However, the ratio for $\left[2 \mathrm{M}_{-} \mathrm{CH}_{3}\right]^{+}$decreases as 194 of 2-propanol from the direct beam to the deflected beam. 176 the position increases in Fig. 3 a and Fig. 3 b, indicating 177 that this peak originates from the dimer or larger clusters, 178 which are deflected out of the original beam, but less than 195 By comparing the intensities of $\left[\mathrm{M}-\mathrm{CH}_{3}\right]^{+}$and $[\mathrm{M}-\mathrm{OH}]^{+}$ 196 in Fig. 3 a and Fig. 3 b, the intensity ratio of the $\mathrm{M} \rightarrow[\mathrm{M}-$ 179 the monomer. $\left.{ }_{197} \mathrm{CH}_{3}\right|^{+}$channel to the $\mathrm{M} \rightarrow[\mathrm{M}-\mathrm{OH}]^{+}$channel was obtained 198 as $\sim 5$ for both peak intensities in the region from $1.1 \mathrm{~mm}$

180 Following the strong-field/multi-photon ionization pro- 199 to $1.6 \mathrm{~mm}$. The fragmentation ratios of the monomer, 181 cess of 2-propanol, $\mathrm{C}-\mathrm{C}$ and $\mathrm{C}-\mathrm{O}$ bonds of the monomer 200 defined as the intensities of the fragments divided by 182 were broken. This suggests that the monomer has two 201 the sum of intensities of the monomer and its related 183 fragmentation channels, $\mathrm{M} \rightarrow\left[\mathrm{M}-\mathrm{CH}_{3}\right]^{+}$and $\mathrm{M} \rightarrow\left[\mathrm{M}-{ }_{202}\right.$ fragments, were estimated as $83 \%$ and $89 \%$ at the lower $\left.{ }_{184} \mathrm{OH}\right]^{+}$.

203 and higher peak intensity, respectively.

185 Based on the intensity of the fragments of the 2- 204 The beam density was estimated based on the analog de186 propanol in the deflected beam, Fig. 2 a and Fig. 2 b, 205 tector current signal calibrated to a single-ion hit. Approx187 the purity of the intact 2-propanol monomer was derived 206 imately 7 ions/shot were created in the deflected beam ${ }_{188}$ as the ratio of the sum of the signals due to $\mathrm{M}^{+}$and $207(+1.4 \mathrm{~mm})$ at a laser peak intensities of $7 \times 10^{13} \mathrm{~W} / \mathrm{cm}^{2}$.

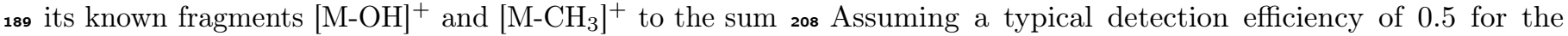
190 of all signals in the mass spectrum. The 2-propanol $209 \mathrm{MCP}$ detector, a molecular-beam width of $1 \mathrm{~mm}$, and a 191 monomer fraction was 4(1) \% in the center of the direct 210 strong-field-ionization probability of 1 , a beam density of 192 beam and 90(4) \% at $+1.4 \mathrm{~mm}$ in the deflected beam. $211 \sim 7 \times 10^{6} \mathrm{~cm}^{-3}$ was obtained for the 2-propanol monomer. 

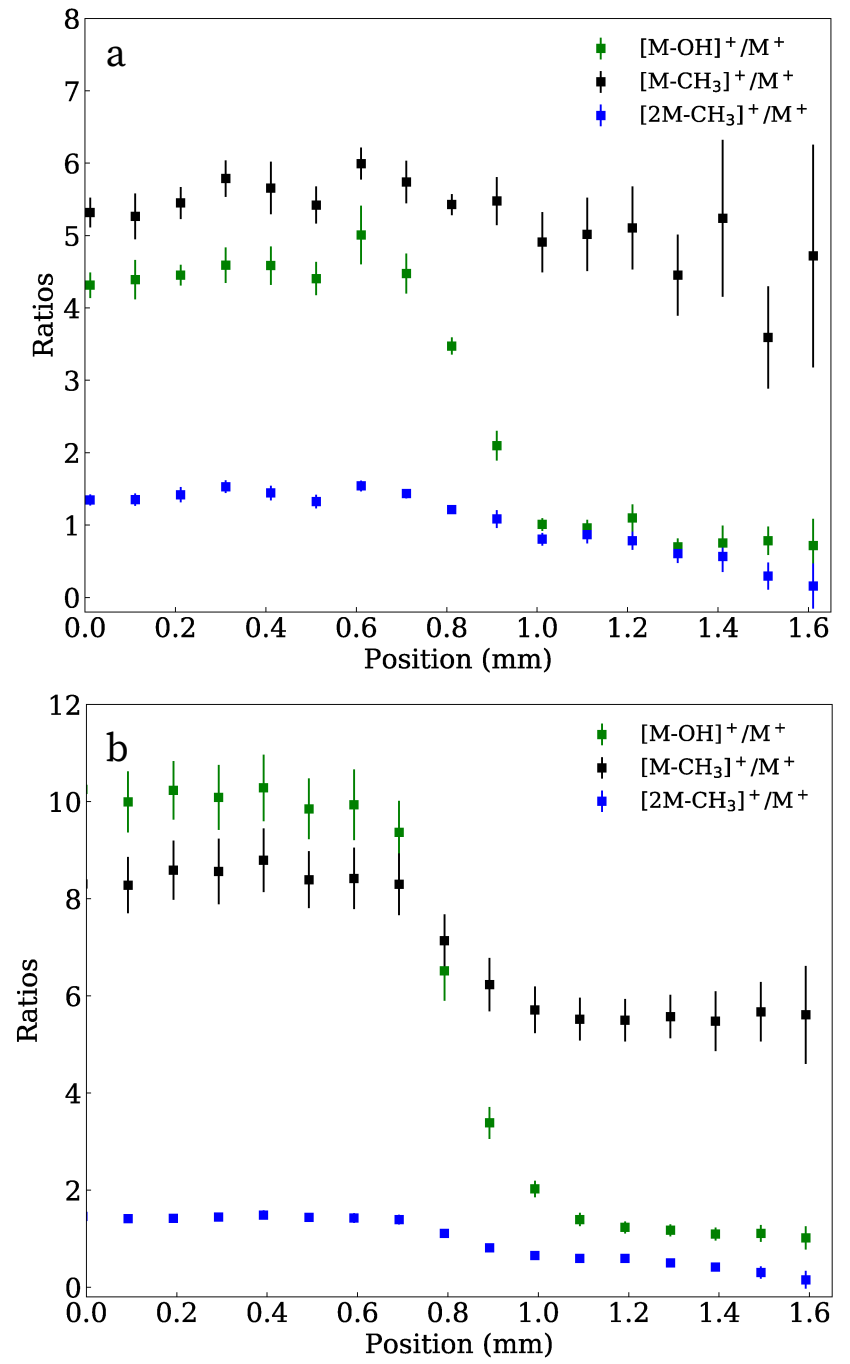

FIG. 3. The ratios of different fragment ions $[\mathrm{M}-\mathrm{OH}]^{+}$(green), $\left[\mathrm{M}-\mathrm{CH}_{3}\right]^{+}$(black), $\left[2 \mathrm{M}-\mathrm{CH}_{3}\right]^{+}$(blue) and $\mathrm{M}^{+}$ion in the deflected region with peak intensities of (a) $3 \times 10^{13} \mathrm{~W} / \mathrm{cm}^{2}$ and (b) $7 \times 10^{13} \mathrm{~W} / \mathrm{cm}^{2}$.

\section{CONCLUSIONS}

${ }_{213}$ A high-purity beam of 2-propanol monomer was pro${ }_{214}$ duced through the spatial separation of the monomer from 215 its clusters and the seed gas using the electrostatic deflec$2 \mathbf{2 1 6}$ tor. The purity and beam density of 2-propanol monomer ${ }_{217}$ were $90(4) \%$ and $7 \times 10^{6} \mathrm{~cm}^{-3}$ in this deflected part of ${ }_{26}^{268}$ $2 \mathbf{2 1}$ the molecular beam. The 45 fs laser-pulse ionization of 270 219 2-propanol with peak intensities of $3 \times 10^{13} \mathrm{~W} / \mathrm{cm}^{2}$ and 271 $2207 \times 10^{13} \mathrm{~W} / \mathrm{cm}^{2}$ was studied. The 2 -propanol monomer ${ }^{272}$ 221 showed two fragmentation channels in the strong-field ${ }^{273}$ 222 ionization process and the ratio of $\mathrm{M} \rightarrow\left[\mathrm{M}-\mathrm{CH}_{3}\right]^{+}$to ${ }_{223} \mathrm{M} \rightarrow[\mathrm{M}-\mathrm{OH}]^{+}$was estimated to be $\sim 5$. The fragmenting 224 fractions of the monomer were estimated to be $83 \%$ and ${ }_{277}$ ${ }_{225}^{22} 89 \%$ at the lower and higher peak intensities, respec- 278
226 tively. The produced intense, cold, and pure 2-propanol 227 monomer beam is well-suited for further investigations, 228 such as diffractive imaging $[26,46]$, chemical reaction $[24]$ 229 and combustion [47] studies.

\section{ACKNOWLEDGMENTS}

231 This work has been supported by the Clusters of Ex232 cellence "Center for Ultrafast Imaging" (CUI, EXC 1074, 233 ID 194651731) and "Advanced Imaging of Matter" (AIM, ${ }_{234}$ EXC 2056, ID 390715994) of the Deutsche Forschungsge235 meinschaft (DFG) and by the European Research Council 236 under the European Union's Seventh Framework Program ${ }_{237}$ (FP7/2007-2013) through the Consolidator Grant COMO238 TION (ERC-Küpper-614507). J.W. and L.H. acknowledge 239 fellowships within the framework of the Helmholtz-OCPC 240 postdoctoral exchange program and J.O. gratefully ac241 knowledges a fellowship by the Alexander von Humboldt 242 Foundation.

| Permanent address: Department of Physics, Tsinghua University, 100084, Beijing, China

$\S$ Permanent address: Institute of Atomic and Molecular Physics, Jilin University, Changchun 130012, China

$\ddagger$ Present address: Department of Physics and Astronomy, University College London, Gower Street, London WC1E 6BT, UK

* Corresponding author. Email: jochen.kuepper@cfel.de URL: https://www.controlled-molecule-imaging.org

[1] A. V. Burenin, Rigorous description of an energy spectrum of the isopropanol molecule taking into account the

$254 \quad$ internal rotation of methyl tops, Opt. Spectrosc. 120,848 255

12 2 A. V. Burenin, A rigorous description of the energy spectrum of the isopropanol molecule taking into account the internal rotation of hydroxyl, Opt. Spectrosc. 120, 854 ${ }_{259}^{258}$ (2016)

260 [3] A. Maeda, I. R. Medvedev, F. C. D. Lucia, and E. Herbst, 261 The millimeter- and submillimeter-wave spectrum of iso262 propanol [(CH3) $2 \mathrm{CHOH}]$, Astrophys. J. Suppl. Ser. 166, $263650(2006)$

4 |4| J. García-Gavín, R. Lissens, A. Timmermans, and A. Goossens, Allergic contact dermatitis caused by isopropyl alcohol: a missed allergen?, Contact Derm. 65, 101 (2011)

5 5 J. C. Dobrowolski, S. Ostrowski, R. Kołos, and M. H. Jamróz, Ar-matrix IR spectra of 2-propanol and its OD, $\mathrm{D}_{7}$ and $\mathrm{D}_{8}$ isotopologues, Vib. Spectrosc. 48, 82 (2008)

[6] C. Tanaka, Vibrational spectra of isopropyl alcohol and its deuterated species $\left(\mathrm{CH}_{3}\right)_{2} \mathrm{CHOD},\left(\mathrm{CD}_{3}\right)_{2} \mathrm{CHOH}$, $\left(\mathrm{CD}_{3}\right)_{2} \mathrm{CHOD}$, Nippon Kagaku Zasshi 83, 521 (1962).

[7] S. Kondo and E. Hirota, Microwave spectrum and internal rotation of isopropyl alcohol, J. Mol. Spectrosc. 34, 97 (1970)

[8] E. Hirota, Internal rotation in isopropyl alcohol studied by microwave spectroscopy, J. Phys. Chem. 83, 1457 (1979) 

291 limited cooling in supersonic expansions, J. Chem. Phys,

${ }_{293}\left[13\right.$ T. Wang, T. Yang, C. Xiao, Z. Sun, D. Zhang, X. Yang, ${ }_{35}$ 294 M. L. Weichman, and D. M. Neumark, Dynamical reso- 358 295 nances in chemical reactions, Chem. Soc. Rev. 47, $6744 \mathbf{3 5 9}$ (2018)

297 14 14 H. Schaal, T. Häber, and M. A. Suhm, Hydrogen bonding 36 298 in 2-propanol. The effect of fluorination, J. Phys. Chem. 362 299 A 104,265(2000) 300 15 15 L. Evangelisti, Q. Gou, G. Feng, W. Caminati, G. J. 364 301 Mead, I. A. Finneran, P. B. Carroll, and G. A. Blake, 365 302 Conformational equilibrium and internal dynamics in the $\mathbf{3 6 6}$ 303 iso-propanol-water dimer, Phys. Chem. Chem. Phys. 19. 367 304 $305[16 \mid$ I. León, I. Usabiaga, J. Millán, E. J. Cocinero, A. Lesarri, 369 306

307 teractions in jets: the propofol-isopropanol cluster, Phys, 308 Chem. Chem. Phys. 16,16968 (2014) 372

зом [17] C. Ng, Molecular beam photoionization studies of $\mathbf{3 7 3}$ molecules and clusters, Adv. Chem. Phys. , 263 (1982) 374

Flowers, Condensation embryos in an expanding gas beam, 376 $313 \quad$ Proc. Royal Soc. London A 280, 409 (1964)

314 [19] G. Fischer, R. E. Miller, P. F. Vohralik, and R. O. Watts, 378 315 Molecular beam infrared spectra of dimers formed from 379

$317 \quad$ source pressure and concentration, J. Chem. Phys. 83.

318 $1471(1985)$

325 22 $\mathrm{H}$. Haberland, A model for the processes happening in 326 a rare-gas cluster after ionization, Surf. Sci. 156, 305 390 327 (1985)

328 [23] W. Schöllkopf and J. P. Toennies, Nondestructive mass 392 329 selection of small van der waals clusters, Science 266, $3 3 0 \longdiv { 1 3 4 5 ( 1 9 9 4 ) }$

331 24] Y.-P. Chang, K. Długołęcki, J. Küpper, D. Rösch, D. Wild, з 332 and $\mathrm{S}$. Willitsch, Specific chemical reactivities of spatially
T. Delmas, S. Epp, B. Erk, L. Foucar, T. Gorkhover L. Gumprecht, A. Hartmann, R. Hartmann, G. Hauser, P. Holl, A. Hömke, N. Kimmel, F. Krasniqi, K.-U. Kühnel, J. Maurer, M. Messerschmidt, R. Moshammer, C. Reich, B. Rudek, R. Santra, I. Schlichting, C. Schmidt, S. Schorb, J. Schulz, H. Soltau, J. C. H. Spence, D. Starodub, L. Strüder, J. Thøgersen, M. J. J. Vrakking, G. Weidenspointner, T. A. White, C. Wunderer, G. Meijer, J. Ullrich, H. Stapelfeldt, D. Rolles, and H. N. Chapman, X-ray diffraction from isolated and strongly aligned gas-phase molecules with a free-electron laser, Phys. Rev. Lett. 112. 083002 (2014) arXiv:1307.4577 [physics]

27] J. Yang, X. Zhu, T. J. A. Wolf, Z. Li, J. P. F. Nunes, R. Coffee, J. P. Cryan, M. Gühr, K. Hegazy, T. F. Heinz, K. Jobe, R. Li, X. Shen, T. Veccione, S. Weathersby, K. J. Wilkin, C. Yoneda, Q. Zheng, T. J. Martínez, M. Centurion, and $\mathrm{X}$. Wang, Imaging $\mathrm{CF}_{3} \mathrm{I}$ conical intersection and photodissociation dynamics with ultrafast electron diffraction, Science 361, 64 (2018)

[28] F. Filsinger, G. Meijer, H. Stapelfeldt, H. Chapman, and J. Küpper, State- and conformer-selected beams of aligned and oriented molecules for ultrafast diffraction studies, Phys. Chem. Chem. Phys. 13, 2076 (2011) arXiv:1009.0871 physics

[29] Y.-P. Chang, D. A. Horke, S. Trippel, and J. Küpper, Spatially-controlled complex molecules and their applications, Int. Rev. Phys. Chem. 34, 557 (2015) arXiv: 1505.05632 [physics]

[30] S. Y. T. van de Meerakker, H. L. Bethlem, N. Vanhaecke, and G. Meijer, Manipulation and control of molecular beams, Chem. Rev. 112, 4828 (2012)

[31] F. Filsinger, J. Küpper, G. Meijer, L. Holmegaard, J. H. Nielsen, I. Nevo, J. L. Hansen, and H. Stapelfeldt, Quantum-state selection, alignment, and orientation of large molecules using static electric and laser fields, J. Chem. Phys. 131, 064309 (2009) arXiv:0903.5413 [physics]

[32] J. H. Nielsen, P. Simesen, C. Z. Bisgaard, H. Stapelfeldt, F. Filsinger, B. Friedrich, G. Meijer, and J. Küpper, Stark-selected beam of ground-state OCS molecules characterized by revivals of impulsive alignment, Phys. Chem. Chem. Phys. 13, 18971 (2011) arXiv:1105.2413 [physics]

33 5 D. A. Horke, Y.-P. Chang, K. Długołęcki, and J. Küpper, Separating para and ortho water, Angew. Chem. Int. Ed. 53, 11965 (2014) arXiv:1407.2056 [physics]

34] F. Filsinger, U. Erlekam, G. von Helden, J. Küpper, and G. Meijer, Selector for structural isomers of neutral molecules, Phys. Rev. Lett. 100, 133003 (2008) arXiv:0802.2795 physics

[35] T. Kierspel, D. A. Horke, Y.-P. Chang, and J. Küpper, Spatially separated polar samples of the cis and trans conformers of 3-fluorophenol, Chem. Phys. Lett. 591, 130 (2014) arXiv:1312.4417 [physics]

36 5 S. Trippel, Y.-P. Chang, S. Stern, T. Mullins, L. Holmegaard, and J. Küpper, Spatial separation of state- and size-selected neutral clusters, Phys. Rev. A 86, 033202 (2012) arXiv:1208.4935 [physics]

37 1 N. Teschmit, D. A. Horke, and J. Küpper, Spatially separating the conformers of a dipeptide, Angew. Chem. Int. Ed. 57, 13775 (2018) arXiv:1805.12396 [physics]

38 1 H. S. You, J. Kim, S. Han, D.-S. Ahn, J. S. Lim, and S. K. Kim, Spatial isolation of conformational isomers of hydroquinone and its water cluster using the stark deflector, J. Phys. Chem. A 122, 1194 (2018) 
407 [39] M. Johny, J. Onvlee, T. Kierspel, H. Bieker, S. Trippel, 427

411

412

413

414

415

416

417

418

419

420

421

422

423

424

425

426

and J. Küpper, Spatial separation of pyrrole and pyrrole- 428 water clusters, Chem. Phys. Lett. 721, 149-152 (2019), arXiv:1901.05267 physics||

0] H. Bieker, J. Onvlee, M. Johny, L. He, T. Kierspel, S. Trippel, D. A. Horke, and J. Küpper, Pure molecular beam 432[ of water dimer, J. Phys. Chem. A 123, 7486 (2019) arXiv:1904.08716 physics

1] D. Irimia, D. Dobrikov, R. Kortekaas, H. Voet, D. A. 435 van den Ende, W. A. Groen, and M. H. M. Janssen, 436 A short pulse $(7 \mu \mathrm{s}$ FWHM) and high repetition rate 437 (dc-5kHz) cantilever piezovalve for pulsed atomic and 438 molecular beams, Rev. Sci. Instrum. 80,113303 (2009) 439

[42] J. S. Kienitz, S. Trippel, T. Mullins, K. Długołęcki, 440 R. González-Férez, and J. Küpper, Adiabatic mixed- 441 field orientation of ground-state-selected carbonyl sul- 442 fide molecules, Chem. Phys. Chem. 17, 3740 (2016) 443 arXiv:1607.05615 [physics

43] S. Trippel, T. Mullins, N. L. M. Müller, J. S. Kienitz, K. Długołęcki, and J. Küpper, Strongly aligned and ori- ented molecular samples at a $\mathrm{kHz}$ repetition rate, Mol. Phys. 111, 1738 (2013) arXiv:1301.1826 [physics]

$44 \mid$ R. S. Ruoff, T. D. Klots, T. Emilsson, and H. S. Gutowsky, Relaxation of conformers and isomers in seeded supersonic jets of inert gases, J. Chem. Phys. 93, 3142 (1990)

[45] Y.-P. Chang, F. Filsinger, B. Sartakov, and J. Küpper, CMIsTaRK: Python package for the stark-effect calculation and symmetry classification of linear, symmetric and asymmetric top wavefunctions in dc electric fields, Comp. Phys. Comm. 185, 339 (2014) arXiv:1308.4076 [physics]

$46]$ A. Barty, J. Küpper, and H. N. Chapman, Molecular imaging using x-ray free-electron lasers, Annu. Rev. Phys. Chem. 64, 415 (2013)

47] C. Esarte, M. Abián, Á. Millera, R. Bilbao, and M. U. Alzueta, Gas and soot products formed in the pyrolysis of acetylene mixed with methanol, ethanol, isopropanol or n-butanol, Energy 43, 37 (2012) 